\title{
AVALIAÇÃO DO EFEITO DA TERMOCICLAGEM NA ESTABILIDADE DE COR E RUGOSIDADE SUPERFICIAL DE DUAS RESINAS COMPOSTAS COMERCIAIS
}

\author{
EVALUATION OF THERMOCYCLING EFFECT ON COLOR STABILITY AND \\ SURFACE ROUGHNESS OF TWO COMMERCIAL RESIN COMPOSITE
}

\author{
Julianne Bartz MAASS'; Luíse dos Santos FERREIRA'; Andressa Goicochea MOREIRA²; Cinthia Studzinski dos SANTOS; \\ Giana da Silveira LIMA4 \\ 1 - Graduação em Odontologia, Faculdade de Odontologia, Universidade Federal de Pelotas (UFPel). \\ 2 - Cirurgiã-Dentista, Mestre e Doutora em Materiais Dentários, Programa de Pós-Graduação em Odontologia, Faculdade de Odontologia, \\ Universidade Federal de Pelotas (UFPel). \\ 3 - Cirurgiã-Dentista, Mestre e Doutoranda em Prótese Dentária, Programa de Pós-Graduação em Odontologia, Faculdade de Odontologia, \\ Universidade Federal de Pelotas (UFPel). \\ 4 - Cirurgiã-Dentista, Mestre e Doutora em Dentística (UFPel), Professora da Faculdade de Odontologia, Departamento de Odontologia \\ Restauradora e do Programa de Pós-Graduação em Odontologia da Universidade Federal de Pelotas (UFPel).
}

\section{RESUMO}

Objetivo: Avaliar o efeito do envelhecimento térmico por termociclagem na estabilidade de cor e rugosidade superficial de duas resinas compostas, avaliar grau de conversão (GC), sorção (SO), solubilidade (SL) e microdureza. Material e método: Foram confeccionados 10 espécimes $(6 \mathrm{~mm} \times 1 \mathrm{~mm})$ para cada resina: Filtek Z350 XT (Z350XT) e Estelite Sigma Quick (ES). Foram avaliados: GC, utilizando um espectrofotômetro no infravermelho, SO e SL. A avaliação da microdureza Vickers (MV) e densidade de ligações cruzadas foi realizada antes e após a imersão em etanol por $24 \mathrm{~h}$. A rugosidade superficial e a estabilidade de cor (CIEDE2000) foram avaliadas antes e após envelhecimento térmico por 2000 ciclos, alternando $30 \mathrm{~s} \mathrm{a} 5^{\circ}, 37^{\circ} \mathrm{e}$ $55^{\circ} \mathrm{C}$. A estabilidade de cor e translucidez foram avaliadas com um espectrofotômetro. Os dados foram submetidos ao teste $\mathrm{t}$ $(\alpha=0,05)$. Resultados: ES apresentou resultados de GC maiores
$(62,5 \% \pm 3,7)$ que Z350XT $(48,7 \% \pm 6,9)(\mathrm{p}=0,004)$. Ambas resinas apresentaram resultados de SO e SL similares. A MV final da ES diminuiu 13\% com relação a inicial $(\mathrm{p}=0,115)$. Z350XT apresentou diminuição de $17 \%(p=0,004)$. ES apresentou aumento de $115 \%$ na rugosidade superficial após a termociclagem $(\mathrm{p}=0,001)$, enquanto a Z350XT apresentou diminuição de 35\% ( $\mathrm{p}=0,299)$. ES apresentou menor alteração de cor $(0,280 \pm 0,093)$, quando comparada a Z350XT $(0,549 \pm 0,183)$. A translucidez foi similar para ambos materiais (ES=30,80; Z350XT=30,35). Conclusão: A termociclagem influenciou na alteração de cor e rugosidade superficial das resinas compostas, demonstrando que podem ser alteradas pela degradação na matriz polimérica.

PALAVRAS-CHAVE: Materiais dentários; Resinas compostas; Cor; Estética dentária; Envelhecimento.

\section{INTRODUÇÃO}

Os compósitos são os materiais de primeira escolha em restaurações diretas ${ }^{1}$. Ao longo do tempo, estes materiais passaram por diversas modificações em sua estrutura, bem como na quantidade de carga inorgânica e matriz orgânica² . A evolução nas propriedades do material em conjunto com o desempenho clínico satisfatório e a possibilidade de preservação da estrutura dentária com o procedimento adesivo, permitem sua ampla indicação e aplicabilidade na prática clínica ${ }^{3}$.

O foco do mercado odontológico atualmente é direcionado aos materiais com propriedades ópticas otimizadas a fim de atender a demanda estética, uma vez que propriedades como cor, brilho, lisura superficial, translucidez e opacidade permitem uma replicação fidedigna das características naturais dos dentes $^{4}$. No entanto, problemas relacionados à longevidade dos tratamentos restauradores diretos ainda são observados e uma das causas de sua substituição está relacionada aos fatores estéticos, uma vez que a estabilidade de cor das restaurações a longo prazo, é um aspecto crítico dos procedimentos restauradores ${ }^{5}$.

Alguns fatores envolvidos no processo de degradação do material restaurador podem ser observados na cavidade oral, como a presença de água que pode degradar compostos, enfraquecendo a matriz polimérica ${ }^{6-8}$. Assim, um efeito deletério sobre a rede polimérica pode ser observado a curto ou a longo prazo, modificando sua estrutura física e quimicamente ${ }^{9}$. A degradação superficial da resina e a lixiviação de material inorgânico podem causar alterações microscópicas, influenciando na rugosidade 
superficial e nas propriedades ópticas do material ${ }^{10}$. Além disso, superfícies rugosas podem gerar prejuízos estéticos e fatores retentivos para placa bacteriana e deposição de pigmentos extrínsecos ${ }^{11}$

Considerando que o ambiente bucal é complexo e envolve mudanças dinâmicas, a simulação do envelhecimento dos materiais na cavidade oral é importante para avaliação de propriedades de materiais odontológicos em laboratório ${ }^{12}$. Alguns protocolos de envelhecimento frequentemente utilizados são a termociclagem, imersão em meios líquidos como etanol ou água e simulação de escovação ${ }^{13}$. O envelhecimento térmico é capaz de reproduzir alterações dinâmicas de tensões e temperatura e assim simular de uma forma mais fiel as condições clínicas ${ }^{14}$.

A avaliação destes parâmetros é importante para predizer o comportamento dos materiais restauradores observando se o efeito do envelhecimento térmico pode interferir nas propriedades físicas e estéticas de um compósito restaurador. Portanto, o objetivo deste estudo foi avaliar o efeito do envelhecimento térmico por termociclagem na estabilidade de cor e rugosidade superficial de duas resinas compostas comerciais, bem como avaliar o grau de conversão, sorção, solubilidade e microdureza dos materiais.

\section{MATERIAL E MÉTODO}

\section{Delineamento experimental}

Neste estudo in vitro foram avaliadas duas resinas compostas: Resina Filtek Z350 (3M ESPE, St. Paul, EUA) (Z350XT) e Estelite Sigma Quick (Tokuyama Dental Corporation, Taitou-ku, Tóquio, Japão) (ES). Para a caracterização inicial dos materiais, foram avaliados: grau de conversão (GC), sorção (SO) e solubilidade (SL). A avaliação da microdureza Vickers (MV) e da densidade de ligações cruzadas foi realizada antes e após a imersão dos espécimes em etanol por $24 \mathrm{~h}$ sob temperatura de $24^{\circ} \mathrm{C}^{15}$. A rugosidade superficial e a alteração de cor foram avaliadas antes e após a termociclagem dos espécimes em uma máquina termocicladora (Thermocycle, Biopdi, São Carlos, SP, Brazil). Os materiais utilizados no estudo e sua composição estão descritos na Tabela 1.

\section{Grau de conversão}

O GC foi avaliado em um aparelho espectrofotômetro infravermelho por Transformada de Fourier (RT-FTIR Shimadzu Prestige-21 Spectrometer, Shimadzu, Tóquio, Japão) equipado com dispositivo de refletância total atenuada, composto por um cristal de diamante, com espelhos de angulação de $45^{\circ}$ (PIKE Technologies, Fitchburg, EUA). Um suporte foi acoplado para a fixação da unidade fotoativadora (Radii Cal, SDI, Bayswater, Victoria, Austrália) ao espectrofotômetro para fotoativação dos materiais. Uma distância uniforme de $5 \mathrm{~mm}$ entre a extremidade da ponteira de fibra ótica e a amostra foi adotada.

Uma porção de cada material foi colocada diretamente sobre o cristal de diamante e uma leitura foi realizada, com o material ainda na forma de monômero. Após esta etapa, o material foi fotopolimerizado por $40 \mathrm{~s}$, tempo recomendado pelo fabricante, e então realizada uma nova leitura, com o material já na forma de polímero. Esse procedimento foi realizado em triplicata para cada uma das resinas. O percentual do GC foi calculado considerando a intensidade da vibração do tipo de estiramento da dupla ligação carbono-carbono $(C=C)$ na frequência de $1635 \mathrm{~cm}^{-116}$.

\section{Preparo dos espécimes}

Foram confeccionados 10 espécimes de cada material (esmalte A2), utilizando uma matriz de silicone redonda (6 $\mathrm{mm} \times 1$ $\mathrm{mm}$ ) para os testes de SO e SL, rugosidade superficial, avaliação da estabilidade de cor e MV. A fotoativação foi realizada com luz de LED (Radii Cal; SDI, Bayswater, Victoria, Austrália) com irradiância de $1200 \mathrm{~mW} / \mathrm{cm}^{2}$ posicionada a $0,5 \mathrm{~mm}$ da superfície durante $40 \mathrm{~s}, 20 \mathrm{~s}$ em cada face da amostra, sobre uma tira de poliéster. Após a polimerização, foi realizado o polimento com lixas de granulação \#2000 (3M, São Paulo, Brasil) apenas nas bordas dos espécimes, pois as superfícies que estavam em contato direto com a tira de poliéster já obtinham lisura superficial uniforme. Os espécimes foram armazenados em água destilada na estufa à $37^{\circ}$ por $24 \mathrm{~h}^{17}$.

\section{Sorção e solubilidade (SO e SL)}

Inicialmente, os espécimes $(\mathrm{n}=10)$ foram colocados em um dessecador de vidro em estufa a $37^{\circ} \mathrm{C}$ por $22 \mathrm{~h}$. Posteriormente, foram transferidos para um segundo dessecador em estufa a $23^{\circ} \mathrm{C}$ por $2 \mathrm{~h}^{18}$. Na sequência, os espécimes foram pesados. Este ciclo foi repetido até que uma massa constante (m1) fosse atingida. Após a obtenção da m1, foi mensurado o diâmetro e a espessura de cada espécime. O diâmetro do espécime foi calculado através da média de dois diâmetros perpendiculares entre si, passando pelo centro do espécime, com um paquímetro digital (Mitutoyo Sul Americana Ltda, São Paulo, Brasil). Para determinar a espessura, o espécime foi mensurado em um ponto central e mais quatro pontos equidistantes na periferia de cada espécime, obtendo-se a média desses cinco valores. Foram então acondicionados em água destilada e armazenado em estufa a $37^{\circ} \mathrm{C}$ por 7 dias $^{18}$. Os espécimes permaneceram suspensos verticalmente, a fim de aumentar a área de contato, em um volume de água de pelo menos $10 \mathrm{ml}$ por espécime. Após 7 dias, foram lavados com água e secos suavemente por $15 \mathrm{~s}$. Então, uma nova mensuração do peso foi obtida (m2), caracterizando assim o peso do espécime mais a água absorvida, medida que mostrou a massa inchada. Após a obtenção da m2, os espécimes voltaram a ser armazenados nos dessecadores, repetindo-se o ciclo inicial, até obter-se uma terceira massa constante chamada de massa seca final (m3). Os espécimes foram pesados em balança analítica/digital (AG200, Gehaka, São Paulo, Brasil) individualmente. Para cada espécime, os dados de $\mathrm{SO}$ e SL em água foram calculados usando as seguintes fórmulas ${ }^{19,20}: \mathrm{SO}=(\mathrm{m} 2-\mathrm{m} 3) / \mathrm{V}$ e $\mathrm{SL}=(\mathrm{m} 1-\mathrm{m} 3) / \mathrm{V}$.

Microdureza vickers (MV) e densidade de ligações cruzadas

A MV $\left(\mathrm{kg} / \mathrm{mm}^{2}\right)$ foi avaliada nos espécimes (MV seca) $(\mathrm{n}=10)$ aplicando uma carga de $50 \mathrm{~g}$ por $15 \mathrm{~s}$ na parte superior da superfície do espécime com o microdurômetro (HMV-2, FM-700, Future Tech Corp, Tóquio, Japão). O comprimento da diagonal de cada endentação foi medido diretamente usando uma lente graduada. O valor da MV foi calculado como anteriormente descrito na literatura ${ }^{21}$. Os mesmos espécimes foram imersos em etanol, por $24 \mathrm{~h}$, sob temperatura de $24^{\circ} \mathrm{C}$, antes de realizar nova aferição da MV (MV etanol). A relação entre MV etanol e MV seca (\%) foi usada para a avaliação da densidade de ligações cruzadas ${ }^{15,22}$. 


\section{Termociclagem}

Após os testes iniciais para avaliação da cor e da rugosidade superficial, os espécimes foram submetidos a 2000 ciclos em banhos alternados de $30 \mathrm{~s}$ nas temperaturas de $5^{\circ}, 37^{\circ}$ e $55^{\circ} \mathrm{C}$ em uma termocicladora (Thermocycle, Biopdi, São Carlos, Brasil) ${ }^{23}$.

\section{Rugosidade superficial}

A rugosidade superficial foi avaliada antes e após a termociclagem, utilizando um rugosímetro digital (Surf-Corder SE 1700, Kosaka Laboratory Ltd., Tóquio, Japão). O aparelho foi calibrado por meio de leitura padrão fornecida pelo fabricante. Na sequência da calibração, cada espécime $(n=10)$ foi acoplado a um dispositivo de leitura, mantido em posição padronizada com um dispositivo de silicone de adição (Adsil, Coltene, Rio de Janeiro, Brasil). O dispositivo foi posicionado perpendicularmente ao espécime, as medidas foram obtidas em Ra e a unidade de medida utilizada foi o micrômetro $(\mu \mathrm{m})$. Foram obtidas três medidas antes e após a termociclagem e então foi determinada a média desses valores para cada espécime.

\section{Avaliação da estabilidade da cor}

Os parâmetros de cor foram mensurados de acordo com as coordenadas individuais CIEDE2000 em uma caixa de calibração em zero $\left(L^{*}=0, a^{*}=0, b^{*}=0, C^{*}=0\right.$ e $\left.h^{\circ}=0\right)$, sob fundo branco $\left(L^{*}=91,7 a^{*}=0,18 b^{*}=-3,85\right)$, sob fundo cinza $(L=45,0 a=-1,11 b=-$ $0,53)$ e sob fundo preto $\left(L^{*}=27,30 a^{*}=0,04 b^{*}=-0,16\right)$ utilizando um espectrofotômetro (SP60, X-Rite, Grand Rapids, EUA) ${ }^{24}$. O equipamento foi calibrado nos padrões fornecidos pelo fabricante. Após o armazenamento dos espécimes $(n=10)$ em água destilada à $37^{\circ} \mathrm{C}$ por $24 \mathrm{~h}$, foi realizada a avaliação inicial. Para avaliar a alteração de cor dos espécimes, uma leitura foi realizada novamente após a termociclagem. $\mathrm{O} \Delta \mathrm{E}$ foi calculado utilizando os valores obtidos sobre o fundo cinza, por meio da fórmula: $\Delta \mathrm{E}_{00}=\left[\left(\Delta \mathrm{L}^{\prime} / \mathrm{k}_{\mathrm{L}} \mathrm{S}_{\mathrm{L}}\right)^{2}+\left(\Delta \mathrm{C}^{\prime} / \mathrm{k}_{\mathrm{C}} \mathrm{S}_{\mathrm{C}}\right)^{2}+\left(\Delta \mathrm{H}^{\prime} / \mathrm{k}_{\mathrm{H}} \mathrm{S}_{\mathrm{H}}\right)^{2}+\mathrm{R}_{\mathrm{T}}\left(\Delta \mathrm{C}^{\prime} / \mathrm{k}_{\mathrm{C}} \mathrm{S}_{\mathrm{C}}\right)\left(\Delta \mathrm{H}^{\prime} /\right.\right.$ $\left.\left.\mathrm{k}_{\mathrm{H}} \mathrm{S}_{\mathrm{H}}\right)\right]^{1 / 2}$. O parâmetro de translucidez (PT) foi calculado utilizando a fórmula ${ }^{25}: \mathrm{PT}=\left[\left(\mathrm{L}_{\mathrm{b}}-\mathrm{L}_{\mathrm{p}}\right)^{2}+\left(\mathrm{a}_{\mathrm{b}}-\mathrm{a}_{\mathrm{p}}\right)^{2}+\left(\mathrm{b}_{\mathrm{b}}-\mathrm{b}_{\mathrm{p}}\right)^{2}\right]^{1 / 2}$, onde $\mathrm{o}$ " $\mathrm{b}$ " subscrito se refere aos valores do CIELAB obtidos sobre o fundo branco e o " $\mathrm{p}$ " subscrito se refere aos valores obtidos sobre fundo preto.

\section{Análise estatística}

Para análise estatística, foi utilizado o software SigmaStat 3.5 (Systat Software, Inc, San Jose, EUA). Os dados foram avaliados quanto à distribuição normal e homogeneidade de variância com base no teste de Shapiro-Wilk. Para cada variável de resposta, os dados foram submetidos a um teste t. Foi adotado um nível de significância de $5 \%$ para todos os testes estatísticos.

\section{RESULTADOS}

Os resultados de GC, SO e SL estão apresentados na Tabela 2. O grupo ES apresentou o GC 22\% mais alto que o grupo Z350XT $(\mathrm{p}=0,004)$. Para os testes de SO (ES=23,04 $\pm 6,71 ; \mathrm{Z350XT}=25,55 \pm$ $3,95)$ e $S L(E S=8,98 \pm 1,92 ; Z 350 X T=8,38 \pm 2,54)$ ambas as resinas avaliadas apresentaram resultados similares.

Na Figura 1 estão apresentados os resultados da densidade de ligações cruzadas, que foi determinada de maneira indireta através da diferença entre a MV final e a MV inicial dos espécimes (\%). O grupo ES apresentou valor de MV inicial de 80,22 $\pm 7,08 \mathrm{~kg} / \mathrm{mm}^{2}$ e Z350XT de 100,56 $\pm 1,68 \mathrm{~kg} / \mathrm{mm}^{2}$. A MV final do grupo ES foi de $69,76 \pm 5,59 \mathrm{~kg} / \mathrm{mm}^{2}$, com diminuição de $13 \%$ (p=0,115). O grupo Z350XT apresentou MV final de $83,54 \pm 4,57 \mathrm{~kg} / \mathrm{mm}^{2}$, com diminuição de $17 \%$ ( $p=0,004)$. A comparação da diminuição de MV entre os materiais não apresentou diferença estatística $(p=0,108)$.

Os valores da rugosidade superficial antes e após a termociclagem podem ser observados na Tabela 3. O grupo Z350XT apresentou uma diminuição de $35 \%$ na rugosidade superficial após a termociclagem $(p=0,299)$. O grupo ES apresentou um aumento na rugosidade final de $115 \%(\mathrm{p}<0,001)$. Embora não tenha sido observada diferença na rugosidade inicial entre os materiais $(\mathrm{p}=0,057)$, houve diferença na rugosidade final $(\mathrm{p}=0,023)$ que foi maior no grupo ES $(0,14 \pm 0,04)$.

Os resultados de alteração de cor e translucidez estão apresentados na Tabela 4. A estabilidade de cor dos espécimes foi comparada antes e após a termociclagem. ES obteve menor alteração de cor $(0,280 \pm 0,093)$ quando comparada à Z350XT $(0,549$ $\pm 0,183)(\mathrm{p}=0,024)$. Entretanto, ambos os valores do $\Delta \mathrm{E}$ foram abaixo do limiar de perceptibilidade $(0,8)$ para o CIEDE200026. Os resultados de translucidez das duas resinas compostas avaliadas foram similares $(E S=30,803$; Z350XT=30,354) $(\mathrm{p}=0,516)$.

Tabela 1 - Composição das resinas avaliadas no estudo, conforme informação dos fabricantes

\begin{tabular}{|c|c|c|}
\hline Materiais & Fabricante /Lote & Composição \\
\hline $\begin{array}{l}\text { Resina Estelite Sigma } \\
\text { Quick A2 }\end{array}$ & $\begin{array}{l}\text { Tokuyama Dental Corporation, } \\
\text { Japão/E690 }\end{array}$ & $\begin{array}{l}\text { 82\% Sílica-Zircônia partículas esféricas <1 micron, Monômeros metacrilatos, bisfenol A, diglicidil, éter, dimetacrilato } \\
\text { (BIS-GMA), Trietileno-Glicol-Dimetacrilato, Iniciador da Fotopolimerização amplificado por radical (tecnologia RAP) e } \\
\text { pigmentos. }\end{array}$ \\
\hline $\begin{array}{l}\text { Resina Filtek } 2350 \\
\text { XT A2 }\end{array}$ & $\begin{array}{l}3 \mathrm{M} \text { ESPE, EUA/ } \\
688062\end{array}$ & $\begin{array}{l}\text { Cerâmica tratada com silano, bisfenol A, diglicidil, éter, dimetacrilato (BIS-GMA), bisfenol A, polietilenoglicol, diéter, di- } \\
\text { metacrilato (BIS-EMA), sílica tratada com silano, sílica-óxido de zircônia tratada com silano, diuretano de dimetacrilato, } \\
\text { dimetacrilato polietilenoglicol, dimetacrilato de trietilenoglicol (TEG-DMA), 2,6-di-terc-butil-t-cresol (BHT) e pigmentos. }\end{array}$ \\
\hline
\end{tabular}

Tabela 2 - Valores médios \pm desvio padrão de Grau de conversão, Sorção e Solubilidade

\begin{tabular}{llll}
\hline Grupos & Grau de conversão $(\%)$ & Sorção $\left(\mu \mathrm{g} / \mathrm{mm}^{3}\right)$ & Solubilidade $\left(\mu \mathrm{g} / \mathrm{mm}^{3}\right)$ \\
\hline ES & $62,55 \pm(3,73)^{\mathrm{A}}$ & $23,04 \pm(6,71)^{\mathrm{A}}$ & $8,98 \pm(1,92)^{\mathrm{A}}$ \\
\hline Z350XT & $48,76 \pm(6,92)^{\mathrm{B}}$ & $25,55 \pm(3,95)^{\mathrm{A}}$ & $8,38 \pm(2,54)^{\mathrm{A}}$ \\
\hline Valor $p$ & 0,004 & 0,635 & 0,759 \\
\hline
\end{tabular}

Letras distintas na mesma coluna representam diferença estatística significante $(p<0,05)$ entre os grupos.
Tabela 3 - Valores médios e desvio padrão da rugosidade superficial (Ra) antes e após termociclagem dos materiais avaliados

\begin{tabular}{llll}
\hline Grupos & Ra Inical $(\mu \mathrm{m})$ & Ra Final $(\mu \mathrm{m})$ & Valor $p$ \\
\hline ES & $0,06( \pm 0,02)^{\mathrm{Aa}}$ & $0,14( \pm 0,04)^{\mathrm{Bb}}$ & 0,001 \\
\hline Z350XT & $0,13( \pm 0,10)^{\mathrm{Aa}}$ & $0,08( \pm 0,04)^{\mathrm{Aa}}$ & 0,299 \\
\hline Valor $p$ & 0,057 & 0,023 & \\
\hline
\end{tabular}

Letras maiúsculas distintas na mesma coluna e letras minúsculas distintas na mesma linha e representam diferença estatística significante $(p<0,05)$ entre os grupos. 


\section{DISCUSSÃO}

Em relação ao GC, neste estudo, Z350XT apresentou um valor $22 \%$ menor que o encontrado para ES. Este resultado pode estar relacionado à diferença de composição dos materiais, pois apesar de ambos apresentarem natureza nanoparticulada, existem diferenças nos componentes da matriz orgânica e nas partículas de carga que podem exercer influência sobre o $\mathrm{GC}^{27}$. O GC possui uma relação direta com as propriedades físico-químicas e a biocompatibilidade do material, pois a baixa conversão pode implicar em redução da resistência mecânica, alteração das propriedades ópticas e da rugosidade superficial do material ${ }^{28}$. Em uma matriz polimérica, os monômeros que não atingem a conversão podem ser lixiviados do material, influenciando sua biocompatibilidade ${ }^{29}$. Embora não exista um valor padrão estabelecido como um GC ideal, valores acima de 55\% são considerados aceitáveis ${ }^{30}$, o que está de acordo com os valores observados para ambos os materiais avaliados neste estudo.

A SL de materiais poliméricos diz respeito à lixiviação de monômeros não reagidos quando em contato com a água e está relacionada ao grau de conversão dos materiais ${ }^{31}$. Quanto maior o GC do material, menor a quantidade de monômeros não reagidos, logo, menor a $\mathrm{SL}^{32}$. A SO significa a capacidade deste material em absorver substâncias do meio, o que pode gerar alterações volumétricas do material ${ }^{33,34}$. Os efeitos destes dois fenômenos podem implicar em prejuízos à estrutura do polímero, influenciando a estabilidade de cor e a rugosidade do material ${ }^{35,36}$. Segundo a norma 4049 da ISO, as resinas compostas

Tabela 4 - Valores médios e desvio padrão para alteração de cor $\left(\Delta \mathrm{E}_{00}\right)$, obtida pela comparação da cor inicial e após a termociclagem e valores de translucidez das duas resinas compostas avaliadas

\begin{tabular}{lll}
\hline Grupos & $\Delta \mathrm{E}_{00}$ & Translucidez \\
\hline ES & $0,280 \pm 0,093^{\mathrm{A}}$ & $30,803^{\mathrm{A}}$ \\
\hline Z350XT & $0,549 \pm 0,183^{\mathrm{B}}$ & $30,354^{\mathrm{A}}$ \\
\hline Valor $p$ & 0,024 & 0,516 \\
\hline
\end{tabular}

Letras maiúsculas distintas na mesma coluna representam diferença estatística significante $(p<0,05)$ entre os grupos.

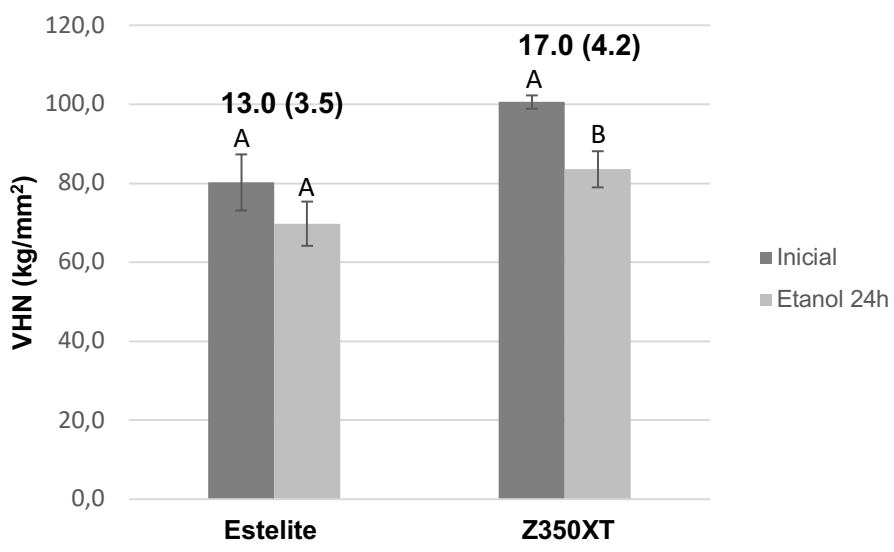

Figura 1 - Gráfico apresentando a densidade de ligações cruzadas com valores médios da microdureza Vickers antes e após imersão em etanol por 24 horas. Redução da microdureza (\%), desvios padrão e resultados estatísticos são exibidos acima das colunas de cada grupo. Não houve diferença estatística na diminuição da dureza entre os grupos. Letras maiúsculas diferentes representam uma diferença estatística entre a microdureza inicial e final do mesmo grupo. devem apresentar SO menor que $40 \mu \mathrm{g} / \mathrm{mm}^{3}$ e SL menor que 7,5 $\mu \mathrm{g} / \mathrm{mm}^{3}$ em um período de armazenamento de 7 dias $^{37}$. No presente estudo, os materiais avaliados apresentaram valores de SL superiores aos recomendados pela ISO. Os valores de SO foram semelhantes entre os grupos, e mais baixos que os valores máximos de SO recomendados, o que demonstra sua reduzida capacidade de absorver água e sofrer expansão volumétrica. Esta característica é desejável para os materiais, uma vez que a cavidade oral é um ambiente úmido e a absorção de água pelo compósito pode levar causar uma deterioração de suas propriedades ${ }^{18,35,38}$.

Nos compósitos, a microdureza está relacionada ao grau de polimerização do material ${ }^{39}$. A profundidade de polimerização é um dos fatores que podem influenciar a diminuição da microdureza do compósito, estando associada à fonte e a intensidade da luz, espectro de emissão do aparelho e tempo e distância de irradiação ${ }^{40}$. Por meio dessa propriedade é possível verificar a resistência superficial do material, quando submetido a algum tipo de deformação ${ }^{41}$. Os altos valores iniciais de MV observados em ambos os materiais (ES=80,22 $\pm 7,08 ; \mathrm{Z350XT}=100,56 \pm 1,68$ ) podem estar associados ao tamanho das partículas presentes na composição dos materiais pois ambas são resinas nanoparticuladas, o que lhes confere boas propriedades mecânicas ${ }^{42}$. No entanto, após 2 mil ciclos de envelhecimento térmico, observou-se uma redução acima de $10 \%$ na microdureza dos materiais, o que está de acordo com resultados reportados para resinas microhíbridas na literatura ${ }^{17}$.

Neste estudo, a porcentagem de densidade de ligações cruzadas foi maior no grupo ES que no grupo Z350XT ( $\mathrm{p}=0,108)$. Essa diferença pode estar associada à diversos fatores tais como: composição da matriz polimérica distinta entre os materiais avaliados; ao GC - que quanto menor, há mais monômeros não reagidos, os quais podem ser lixiviados quando expostos à imersão em etanol ${ }^{43}$; presença de monômeros de alto peso molecular, que tendem a formar ciclos ao invés de cadeias reticuladas, resultando em monômeros de baixo peso molecular que podem ser facilmente lixiviados ${ }^{44}$. Materiais poliméricos podem apresentar ligações cruzadas diferentes por possuírem comprimentos de cadeias distintos ${ }^{45}$. Polímeros com rede reticulada em sua cadeia polimérica são considerados mais resistentes à degradação de solventes orgânicos, enquanto polímeros lineares apresentam maior propensão à propagação dos solventes dentro da sua estrutura $^{46,47}$. Se o solvente penetrar na rede polimérica, pode causar a liberação de monômeros residuais e consequente dissolução linear da estrutura ${ }^{48}$.

A rugosidade superficial dos materiais, inicialmente, apresentou-se semelhante. No entanto, após a termociclagem, a rugosidade do grupo ES aumentou significantemente enquanto que a rugosidade da Z350XT diminuiu $(\mathrm{p}=0,023)$, embora alguns estudos na literatura não tenham observado influência da termociclagem na rugosidade superficial de resinas compostas ${ }^{49,50}$. O envelhecimento térmico pode gerar tensões na estrutura da resina composta, devido às diferenças no coeficiente de expansão térmica linear da matriz orgânica e dos componentes de carga, levando à degradação e possíveis microfissuras superficiais, o que pode justificar o aumento na rugosidade da $\mathrm{ES}^{51}$. Além disso, o aumento da rugosidade pode implicar em maior colonização bacteriana da superfície, quando comparada às superfícies lisas e polidas, favorecendo acúmulo de biofilme na restauraçãa $0^{52}$. 
Com a utilização de 2 mil ciclos de termociclagem, já foi possível observar uma influência na estabilidade de cor dos materiais avaliados, apesar dos valores terem ficado abaixo do limiar considerado perceptível clinicamente ${ }^{26}$. O envelhecimento térmico, aplicado neste estudo por meio da termociclagem, é capaz de reproduzir alterações dinâmicas de tensões e temperatura a fim de simular às condições da cavidade oral ${ }^{53,54}$. O grupo Z350XT demonstrou maior alteração de cor comparado ao grupo ES. Esse resultado pode ser explicado pelas características da composição das resinas compostas como diferenças na matriz polimérica, dimensões das partículas de carga e hidrofilicidade dos materiais ${ }^{55,56}$. A absorção de água tem a capacidade de alterar o padrão de difusão de luz, pois possui índice de refração diferente da rede polimérica e das partículas de carga ${ }^{57}$. Embora as resinas avaliadas possuam uma composição semelhante, a resina Z350XT possui alta porcentagem de TEGDMA o que a torna mais suscetível a descoloração comparada à resina ES e também à Filtek Z250 como relatado anteriormente na literatura ${ }^{58}$.

Neste estudo, os resultados de translucidez das resinas compostas avaliadas foram semelhantes. A translucidez é uma propriedade óptica que pode influenciar a obtenção da cor adequada e consequentemente na estética, uma vez que materiais muito translúcidos podem causar a visualização de uma tonalidade acinzentada da restauração em comparação aos dentes hígidos adjacentes ${ }^{59,60}$. Alterações na translucidez após a polimerização e envelhecimento também foram observadas anteriormente na literatura ${ }^{61}$.

Considerando as limitações inerentes à realização de um estudo in vitro, é importante ressaltar que os resultados desse estudo não devem ser extrapolados para o contexto clínico, no entanto, a avaliação destes parâmetros é importante para predizer o comportamento dos materiais restauradores. Além disso, um maior tempo (ciclos) de envelhecimento térmico seria interessante para obter resultados mais fidedignos.

\section{CONCLUSÕES}

Com base nos resultados deste estudo, pode-se concluir que:

a. Na caracterização das resinas compostas, Z350XT apresentou um GC inferior. Ambas apresentaram resultados similares de SO e SL, além de diminuição da MV após envelhecimento em etanol.

b. O envelhecimento térmico pela termociclagem influenciou a estabilidade de cor e a rugosidade superficial das resinas compostas avaliadas, demonstrando que o material pode sofrer degradação em sua matriz polimérica ao longo do tempo, alterando suas propriedades físicas. Embora ambos os valores tenham ficado abaixo do limiar de perceptibilidade clínica, Z350XT apresentou maior alteração de cor.

\section{AGRADECIMENTOS}

O presente trabalho foi realizado com apoio da Coordenação de Aperfeiçoamento de Pessoal de Nível Superior - Brasil (CAPES) - Código de Financiamento 001.

\section{REFERÊNCIAS}

01. Demarco FF, Corrêa MB, Cenci MS, Moraes RR, Opdam NJM. Longevity of posterior composite restorations: not only a matter of materials. Dent Mater. 2012; 28(1): 87-101.
02. Soares PV, Peres TS, Wobido AR, Machado AC. Composite resin in the last 10 years: literature review. Part 1: chemical composition. J Clin Dent Res. 2019; 16(1): 45-56.

03. Ferracane JL. Resin composite: state of the art. Dent Mater. 2011; 27(1): 29-38.

04. Romero MF. Esthetic anterior composite resin restorations using a single shade: Step-by-step technique. J Prosthet Dent. 2015; 114(1): 9-12.

05. Oliveira DCRS, Souza-Júnior EJ, Prieto LT, Coppini EK, Maia RR, Paulillo LAMS. Color stability and polymerization behavior of direct esthetic restorations. J Esthet Restor Dent. 2014; 26(4): 288-95.

06. Martos J, Osinaga PWR, Oliveira E, Castro LAS. Hydrolytic degradation of composite resins: effects on the microhardness. Mater Res. 2003; 6(4): 599-604.

07. Ferracane JL, Berge HX, Condon JR. In vitro aging of dental composites in water: effect of degree of conversion, filler volume, and filler/matrix coupling. J Biomed Mater Res. 1998; 42(3): 465-72.

08. Delaviz Y, Finer Y, Santerre JP. Biodegradation of resin composites and adhesives by oral bacteria and saliva: A rationale for new material designs that consider the clinical environment and treatment challenges. Dent Mater. 2014; 30(1): 16-32.

09. Ricci WA, Alfano P, Pamato S, Cruz CADS, Pereira JR. Mechanical degradation of different classes of composite resins aged in water, air, and oil. Biomed Res Int. 2019;2019.

10. Maran BM, Naufel FS, Paula AB, Araújo GSA, Puppin-Rontani RM. Biological and mechanical degradation affecting the surface properties of aesthetic restorative. Brazilian J Oral Sci. 2018; 16: 1-10.

11. Moraes RR, Marimon JLM, Schneider LF, Sinhoreti MAC, CorrerSobrinho L, Bueno M. Effects of 6 months of aging in water on hardness and surface roughness of two microhybrid dental composites. J Prosthodont. 2008; 17(4): 323-6.

12. Szczesio-Wlodarczyk A, Sokolowski J, Kleczewska J, Bociong K. Ageing of dental composites based on methacrylate resins: a critical review of the causes and method of assessment. Polymers (Basel). 2020; 12(4): 882.

13. Rocha RS, Oliveira AC, Caneppele TMF, Bresciani E. Effect of Artificial Aging Protocols on Surface Gloss of Resin Composites. Int J Dent. 2017; 2017.

14. Morresi AL, D'Amario M, Capogreco M, Gatto R, Marzo G, $\mathrm{D}^{\prime}$ Arcangelo $\mathrm{C}$, et al. Thermal cycling for restorative materials: does a standardized protocol exist in laboratory testing? a literature review. J Mech Behav Biomed Mater. 2014; 29: 295-308.

15. Leprince JG, Palin WM, Vanacker J, Sabbagh J, Devaux J, Leloup G. Physico-mechanical characteristics of commercially available bulkfill composites. J Dent. 2014; 42(8): 993-1000.

16. Moraes RR, Faria-e-Silva AL, Ogliari FA, Correr-Sobrinho L, Demarco FF, Piva E. Impact of immediate and delayed light activation on self-polymerization of dual-cured dental resin luting agents. Acta Biomater. 2009; 5(6): 2095-100.

17. Ghavami-Lahiji M, Firouzmanesh M, Bagheri H, Jafarzadeh Kashi TS, Razazpour F, Behroozibakhsh M. The effect of thermocycling on the degree of conversion and mechanical properties of a microhybrid dental resin composite. Restor Dent Endod. 2018; 43(2): e26.

18. Berger SB, Palialol ARM, Cavalli V, Giannini M. Characterization of water sorption, solubility and filler particles of light-cured composite resins. Braz Dent J. 2009; 20(4): 314-8.

19. Melo RA, Bispo ASL, Barbosa GAS, Galvão MR, Assunção IV., Souza ROA, et al. Morphochemical characterization, microhardness, water sorption, and solubility of regular viscosity bulk fill and traditional composite resins. Microsc Res Tech. 2019; 1-7. 
20. Pieper CM, Zanchi CH, Rodrigues-Junior SA, Moraes RR, Pontes LS, Bueno M. Sealing ability, water sorption, solubility and toothbrushing abrasion resistance of temporary filling materials. Int Endod J. 2009; 42(10): 893-9.

21. Leprince J, Palin WM, Mullier T, Devaux J, Vreven J, Leloup G. Investigating filler morphology and mechanical properties of new low-shrinkage resin composite types. J Oral Rehabil. 2010; 37(5): 364-76.

22. Feitosa VP, Fugolin APP, Correr AB, Correr-Sobrinho L, Consani S, Watson TF, et al. Effects of different photo-polymerization protocols on resin-dentine $\mu \mathrm{tBS}$, mechanical properties and cross-link density of a nano-filled resin composite. J Dent. 2012; 40(10): 802-9.

23. Consani S, Sinhoreti MAC, Correr-Sobrinho L, Leite RSB, Bortoletto KJ. An alternative method for thermal cycling test: Effect on the marginal microleakage and bond strength of dental polymer bonded to dentin. Mater Res. 2012; 15(6): 1045-9.

24. Sharma G, Wu W, Dalal EN. The CIEDE2000 color-difference formula: implementation notes, supplementary test data, and mathematical observations. Color Res Appl. 2005; 30(1): 21-30.

25. Perroni AP, Amaral C, Kaizer MR, Moraes RR, Boscato N. Shade of resin-based luting agents and final color of porcelain veneers. J Esthet Restor Dent. 2016; 28(5): 295-303.

26. Paravina RD, Ghinea R, Herrera LJ, Bona AD, Igiel C, Linninger M, et al. Color difference thresholds in dentistry. J Esthet Restor Dent. 2015; 27(S1): S1-9.

27. Rodrigues SA, Zanchi CH, Carvalho RV, Demarco FF. Flexural strength and modulus of elasticity of different types of resin-based composites. Braz Oral Res. 2007; 21(1): 16-21.

28. Ferracane JL, Mitchem JC, Condon JR, Todd R. Wear and marginal breakdown of composites with various degrees of cure. J Dent Res. 1997; 76(8): 1508-16.

29. Ak AT, Alpoz AR, Bayraktar O, Ertugrul F. Monomer Release from Resin Based Dental Materials Cured With LED and Halogen Lights. Eur J Dent. 2010; 4(1): 34-40.

30. Alshali RZ, Silikas N, Satterthwaite JD. Degree of conversion of bulkfill compared to conventional resin-composites at two time intervals. Dent Mater. 2013; 29(9): e213-7.

31. Silva EM, Almeida GS, Poskus LT, Guimarães JGA. Relationship between the degree of conversion, solubility and salivary sorption of a hybrid and a nanofilled resin composite: Influence of the lightactivation mode. J Appl Oral Sci. 2008; 16(2): 161-6.

32. Gonçalves L, Filho JDN, Guimarães JGA, Poskus LT, Silva EM. Solubility, salivary sorption and degree of conversion of dimethacrylate-based polymeric matrixes. J Biomed Mater Res - Part B Appl Biomater. 2008; 85b(2): 320-325.

33. Toledano M, Osorio R, Osorio E, Fuentes V, Prati C, García-Godoy F. Sorption and solubility of resin-based restorative dental materials. J Dent. 2003; 31(1): 43-50.

34. Sideridou ID, Karabela MM, Vouvoudi EC. Volumetric dimensional changes of dental light-cured dimethacrylate resins after sorption of water or ethanol. Dent Mater. 2008; 24(8):1131-6.

35. Mohamed EL-Sharkawy F, Mohamed Zaghloul N, Mohsen Ellkappaney A. Effect of Water Absorption on Color Stability of Different Resin Based Restorative Materials in Vitro Study. Int J Compos Mater. 2012; 2(2): 7-10.

36. Hamouda IM. Effects of various beverages on hardness, roughness, and solubility of esthetic restorative materials. J Esthet Restor Dent. 2011; 23(5): 315-22
37. International Organization for Standardization. Dentistry: polymerbased filling, restorative and luting materials. ISO 4049. 2009.

38. Söderholm KJ, Zigan M, Ragan M, Fischlschweiger W, Bergman M. Hydrolytic degradation of dental composites. J Dent Res. 1984; 63(10): 1248-54.

39. Santini A, Miletic V, Swift MD, Bradley M. Degree of conversion and microhardness of TPO-containing resin-based composites cured by polywave and monowave LED units. J Dent. 2012; 40(7): 577-84.

40. Groninger AIS, Soares GP, Sasaki RT, Ambrosano GMB, Lovadino JR, Aguiar FHB. Microhardness of nanofilled composite resin lightcured by LED or QTH units with different times. Brazilian J Oral Sci. 2011; 10(3): 189-92.

41. Wang L, D’Alpino PHP, Lopes LG, Pereira JC. Mechanical properties of dental restorative materials: relative contribution of laboratory tests. J Appl Oral Sci. 2003; 11(3): 162-7.

42. Hamouda IM, Elkader HA. Evaluation the mechanical properties of nanofilled composite resin restorative material. J Biomater Nanobiotechnol. 2012; 3(2): 238-42.

43. Schneider LFJ, Moraes RR, Cavalcante LM, Sinhoreti MAC, CorrerSobrinho L, Consani S. Cross-link density evaluation through softening tests: Effect of ethanol concentration. Dent Mater. 2008; 24(2): 199-203.

44. Podgórski M. Synthesis and characterization of novel dimethacrylates of different chain lengths as possible dental resins. Dent Mater. 2010; 26(6): e188-94.

45. Nakka JS, Jansen KMB, Ernst LJ. Effect of chain flexibility in the network structure on the viscoelasticity of epoxy thermosets. J Polym Res. 2011;18: 1879-1888.

46. Ferracane JL. Hygroscopic and hydrolytic effects in dental polymer networks. Dent Mater. 2006; 22(3): 211-22.

47. Palin WM, Fleming GJP, Burke FJT, Marquis PM, Randall RC. The influence of short and medium-term water immersion on the hydrolytic stability of novel low-shrink dental composites. Dent Mater. 2005; 21(9): 852-63.

48. Ferracane JL. Elution of leachable components from composites. J Oral Rehabil. 1994; 21(4): 441-52.

49. Tuncer S, Demirci M, Tiryaki M, Ünlü N, Uysal Ö. The effect of a modeling resin and thermocycling on the surface hardness, roughness, and color of different resin composites. J Esthet Restor Dent. 2013; 25(6): 404-19.

50. Pala K, Tekçe N, Tuncer S, Serim ME, Demirci M. Evaluation of the surface hardness, roughness, gloss and color of composites after different finishing/polishing treatments and thermocycling using a multitechnique approach. Dent Mater J. 2016; 35(2): 278-89.

51. Rinastiti M, Özcan M, Siswomihardjo W, Busscher HJ. Effects of surface conditioning on repair bond strengths of non-aged and aged microhybrid, nanohybrid, and nanofilled composite resins. Clin Oral Investig. 2011; 15(5): 625-33.

52. Park JW, Song CW, Jung JH, Ahn SJ, Ferracane JL. The effects of surface roughness of composite resin on biofilm formation of Streptococcus mutans in the presence of saliva. Oper Dent. 2012; 37(5): 532-9.

53. Lee YK, Lim BS, Rhee SH, Yang HC, Powers JM. Changes of optical properties of dental nano-filled resin composites after curing and thermocycling. J Biomed Mater Res - Part B Appl Biomater. 2004; 71(1): $16-21$.

54. Minami H, Hori S, Kurashige H, Murahara S, Muraguchi K, Minesaki $\mathrm{Y}$, et al. Effects of thermal cycling on surface texture of restorative composite materials. Dent Mater J. 2007; 26(3): 316-22. 
55. Lee YK, Lim BS, Rhee SH, Yang HC, Powers JM. Color and translucency of A2 shade resin composites after curing, polishing and thermocycling. Oper Dent. 2005; 30(4): 436-42.

56. Vichi A, Ferrari M, Davidson CL. Color and opacity variations in three different resin-based composite products after water aging. Dent Mater. 2004; 20(6): 530-4.

57. Salgado VE, Cavalcante LM, Moraes RR, Davis HB, Ferracane JL, Schneider LF. Degradation of optical and surface properties of resinbased composites with distinct nanoparticle sizes but equivalent surface area. J Dent. 2017; 59: 48-53.
58. Kim HJ, Kim M, Song B, Kim S, Kim J. Effect of polishing methods on color change by water absorption in several composite resins. J Dent Rehabil Appl Sci. 2019; 35(1): 1-10.

59. Piccoli YB, Lima VP, Basso GR, Salgado VE, Lima GS, Moraes RR. Optical stability of high-translucency resin-based composites. Oper Dent. 2019; 44(5): 536-44.

60. Ikeda T, Murata Y, Sano H. Translucency of opaque-shade resin composites. Am J Dent. 2004; 17(2): 127-30.

61. Lee YK. Translucency changes of direct esthetic restorative materials after curing, aging and treatment. Restor Dent Endod. 2016; 41(4): 239-245.

\section{ABSTRACT}

Objective: To evaluate the thermocycling effect on color stability and surface roughness of two resin composites and also evaluate the degree of conversion (GC), sorption (SO), solubility (SL) and microhardness. Material and method: 10 specimens (6 $\mathrm{mm} \times 1 \mathrm{~mm}$ ) were made for each resin: Filtek Z350 XT (Z350XT) and Estelite Sigma Quick (ES). The following were evaluated: GC, using an infrared spectroscopy, SO and SL. Vickers microhardness (MV) and cross-link density evaluation was performed before and after immersion in ethanol for 24 hours. Surface roughness and color stability (CIEDE2000) were evaluated before and after thermal aging for 2000 cycles, alternating between $30 \mathrm{~s}$ at $5^{\circ}, 37^{\circ}$ and $55^{\circ} \mathrm{C}$. Color stability and translucency were assessed with a spectrophotometer. Data were submitted to $t$ test $(\alpha=0.05)$. Results: ES presented higher GC results $(62.5 \%$ \pm 3.7) than Z350XT $(48.7 \% \pm 6.9)(\mathrm{p}=0.004)$. Both resins showed similar SO and SL results. The final MV of ES decreased $13 \%$ in relation to the initial $(p=0.115)$. Z350XT decreased by $17 \%(p=$ 0.004 ). ES showed a $115 \%$ increase in surface roughness after thermocycling $(\mathrm{p}=0.001)$, while the Z350XT showed a 35\% decrease $(p=0.299)$. ES showed less color change $(0.280 \pm 0.093)$ when compared to Z350XT $(0.549 \pm 0.183)$. The translucency was similar for both materials (ES=30.80; Z350XT=30.35). Conclusion: Thermocycling influenced the change in color stability and surface roughness of composite resins, demonstrating that they may be altered by degradation in the polymer matrix.

KEYWORDS: Dental materials; Composite resins; Color; Dental esthetics; Aging.

\section{AUTOR PARA CORRESPONDÊNCIA}

Giana da Silveira Lima

Programa de Pós-Graduação em Odontologia, Faculdade de

Odontologia, Universidade Federal de Pelotas.

Rua Gonçalves Chaves, 457, Pelotas, Rio Grande do Sul,

Brasil. CEP: 96015-560.

Telefone: +55 (53) 999522626.

E-mail: gianalima@gmail.com 